\title{
HRTEM and EELS Studies on the Structural and Chemical Modification of MoS2 and Graphite During In-situ Reactions with $\mathrm{Li}$ and Na
}

\author{
Chanchal Ghosh $^{1}$, Manish Singh ${ }^{1}$, Matthew Janish ${ }^{1}$, Shayani Parida ${ }^{1}$, Avinash M Dongare ${ }^{1}$, C Barry \\ Carter $^{1,2}$ \\ ${ }^{1}$ University of Connecticut, Storrs, Connecticut, United States, ${ }^{2}$ Center for Integrated Nanotechnologies \\ (CINT), Sandia National Laboratories, Albuquerque, New Mexico, United States
}

2D metal sulphides are receiving attention due to their potential application as cathode materials in Lithium (Li) and Sodium (Na) ion batteries [1]; they are compared with graphite which is currently widely used for this purpose. Due to weak van der Waals interaction, these materials can accommodate reversible insertion and retraction of $\mathrm{Li}$ and $\mathrm{Na}$ with the application of suitable biasing. In the family of 2D transition metal dichalcogenides (TMDCs), $\mathrm{MoS}_{2}$ is a most promising material due to its intermediate insertion voltage, high reversible capacity and excellent rate capability [2-3]. Reactions between $\mathrm{MoS}_{2}$ with Li and $\mathrm{Na}$ inside a TEM [4] are studied in order to explore the functionality of this material for cathode application. Reports on possible phase transformations and the structural modification of $\mathrm{MoS}_{2}$ after the solid-state reaction with $\mathrm{Li}$ and $\mathrm{Na}$ still leave many open questions. In-situ and operando reactions involving intercalation of 2D materials have challenges which are documented in the literature [5]. In the present paper, changes in microstructure and microchemistry during in-situ reaction are discussed including the formation of new phases.

In this study, $\mathrm{MoS}_{2}$ and graphite have been mechanically exfoliated using adhesive tape and then immersed in acetone. The exfoliated flakes are collected in a $\mathrm{Cu}$ half grid and then loaded in Nanofactory TEM-STM holder. The Li/Na is loaded in a tungsten probe in Argon atmosphere inside glovebox. In-situ reactions have been carried out in a Tecnai F30 (S)TEM equipped with a Fischione HAADF detector and a GIF for EELS. The post-lithiated specimen were further studied in image-corrected Titan for local structural changes. Image simulation for structural identification were carried out using Java based EMS (JEMS) software [6].

Selected-area diffraction patterns (SAD), bright field images and HRTEM images from the layer materials before reaction with $\mathrm{Li}$ were recorded for comparison with the lithiated microstructure. The studies are illustrated below for $\mathrm{MoS}_{2}$. The SAD patterns confirm the structure as the [101] zone-axis pattern of $\mathrm{MoS}_{2}$ as shown in Fig.1(a). The corresponding electron energy-loss spectroscopy (EELS) profile of S-L (165 $\mathrm{eV})$ and Mo-M (227 eV) is shown in Fig 2. The nature of the ELNES profile of Mo and S are compared with those in the EELS Atlas and match satisfactorily. In similar chronology, the diffraction contrast imaging, phase contrast imaging and the EELS characterization of the Li were also carried out for further comparison after lithiation. The in-situ reaction with $\mathrm{Li}$ inside the TEM were carried out after applying a bias of $-2 \mathrm{~V}$. The propagation of the reaction front of Li with $\mathrm{MoS}_{2}$ in its planar geometry was observed in real time. The changes in the $\mathrm{MoS}_{2}$ after interacting with Li have been analyzed with diffraction contrast and phase contrast imaging as well as the localized chemistry has been determined with the aid of EELS, EFTEM and STEM coupled spectroscopic methods.

The presence of additional spots in the SAD pattern is consistent with the formation of new phases in $\mathrm{MoS}_{2}$ lattice after interacting with $\mathrm{Li}$ as shown in Fig 1(b). Formation of a band like morphology is observed along the reaction front of $\mathrm{MoS}_{2}$ with Li and has been analyzed further with HRTEM and EELS. 
Multislice image simulations using imaging conditions corresponding to the Tecnai F30 and the Titan were carried out with JEMS [6] using structures derived using DFT calculations of different models, and compared with the experimental results. Similar experiments have also been carried out to study the insitu reaction of $\mathrm{MoS}_{2}$ with $\mathrm{Na}$ and the microstructural and the microchemical analysis of the newly formed phases will be discussed.

This research is funded by NSF under award DMR-1820565. TEM investigation was carried out at CINT, an Office of Science User Facility operated for the U.S. DOE, and in the Materials Characterization Department. Sandia National Labs is managed and operated by National Technology and Engineering Solutions of Sandia, LLC., a wholly owned subsidiary of Honeywell International, Inc., for the U.S. DOE's NNSA contract DE-NA-0003525. The views expressed here do not necessarily represent the views of the U.S. DOE or the U.S. Government. Matt Janish is now a member of staff at Los Alamos Laboratories.

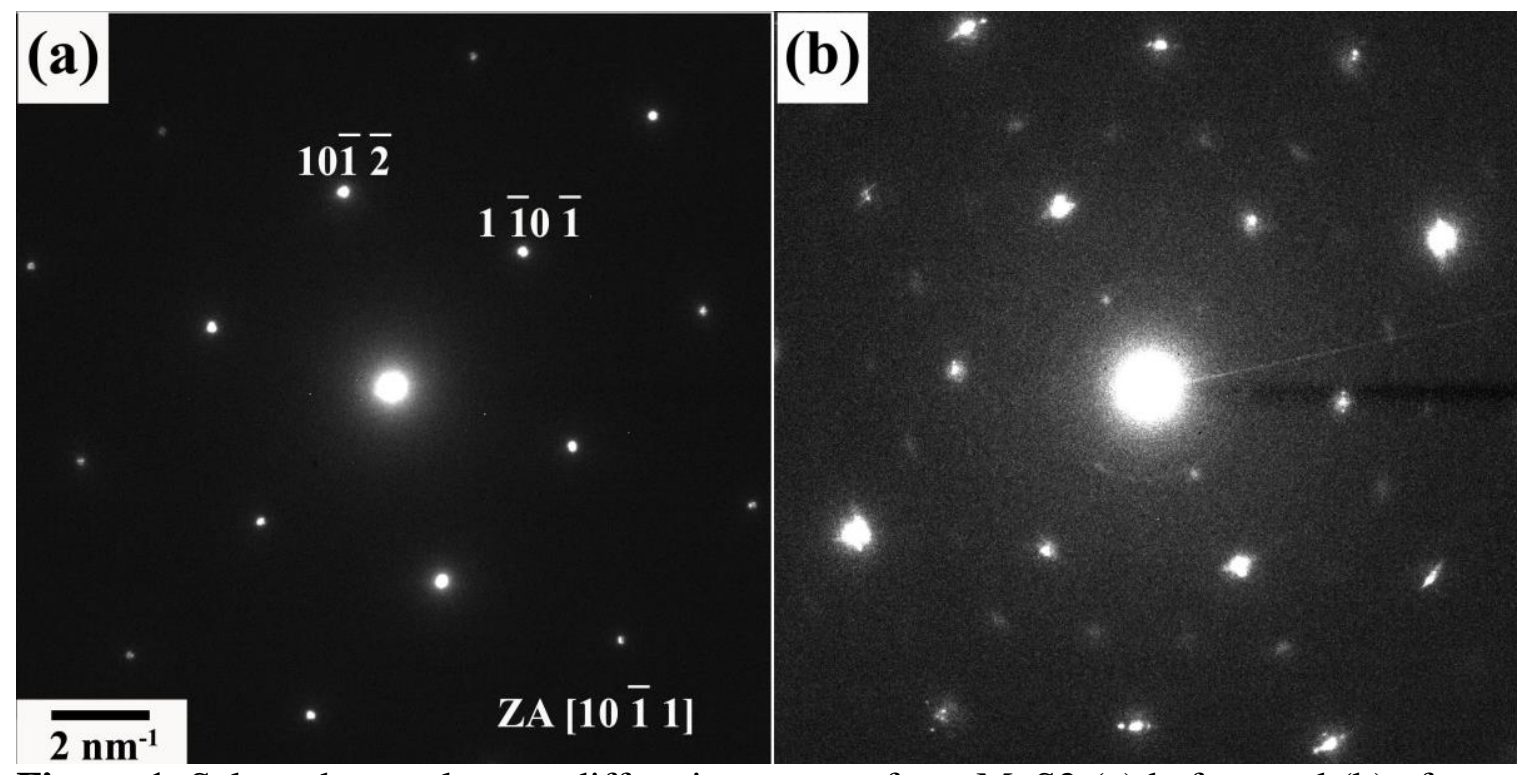

Figure 1. Selected area electron diffraction pattern from MoS2 (a) before and (b) after reacting with Li. The presence of additional spots in (b) signifies the formation of new phases.

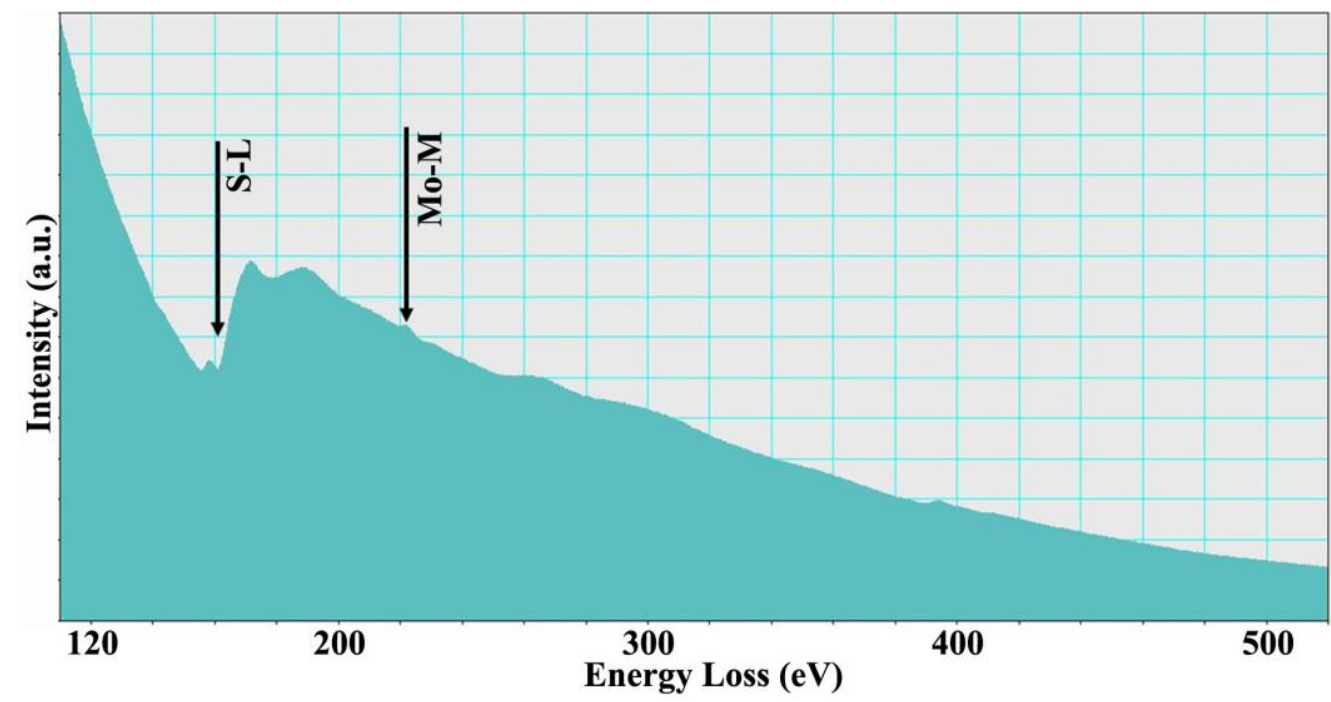


Figure 2. Electron energy loss profile from Mo and S before reaction with Li. The nature of ELNES for both varies significantly after reaction.

\section{References}

[1] Whittingham MS (2004) Lithium batteries and cathode materials, Chem Rev 104, 4271-4301

[2] Janish MT, Carter CB (2015) In situ observations of the lithiation of molybdenum disulfide. Scripta Mater 107, 22-25

[3] Chen S, Wang, Shao R et al (2018) Atomic structure and migration dynamics of $\mathrm{MoS}_{2} / \mathrm{Li}_{\mathrm{x}} \mathrm{MoS}_{2}$ interface. Nano Energy 48, 560-568

[4] Cheng Y, Nie A, Zhang Q et al (2014) Origin of the phase transition in lithiated molybdenum disulphide. ACS Nano 8, 11447-11453

[5] Carter CB, Williams DB (Eds.) (2016) Transmission electron microscopy: Diffraction, Imaging and Spectrometry, Springer, Berlin Heidelberg, 17-80

[6] Stadelmann P (1987) EMS - a software package for electron diffraction analysis and HREM image simulation in materials science. Ultramicroscopy 21(2), 131-145 